Supporting information for

\title{
Facile Arm-first Synthesis of Star Block Copolymers via ARGET ATRP with ppm Amounts of Catalyst
}

Hangjun Ding, ${ }^{\mathrm{a}, \mathrm{b}, \dagger}$ Sangwoo Park, ${ }^{\mathrm{a} \dagger}$ Mingjiang Zhong,,${ }^{\mathrm{a}, \mathrm{l}}$ Xiangcheng Pan, ${ }^{\mathrm{a}}$ Joanna Pietrasik, ${ }^{\mathrm{a}, \mathrm{c}}$ Christopher John Bettinger ${ }^{\text {bd, }}$, and Krzysztof Matyjaszewski, ${ }^{\text {a, }}$

aDepartment of Chemistry, Carnegie Mellon University, 5000 Forbes Avenue, Pittsburgh, PA 15213, United States of America

${ }^{b}$ Department of Materials Science and Engineering, Carnegie Mellon University, 5000 Forbes Avenue, Pittsburgh, PA 15213, United States of America

'Institute of Polymer and Dye Technology, Lodz University of Technology, Stefanowskiego 12/16, 90-924 Lodz, Poland

${ }^{\mathrm{d} D e p a r t m e n t}$ of Biomedical Engineering, Carnegie Mellon University, 5000 Forbes Avenue, Pittsburgh, PA 15213, United States of America

'Present Address: Department of Chemical and Environmental Engineering, Yale University, 17 Hillhouse Ave, New Haven, CT 06511

†These authors contribute equally.

*To whom correspondence should be addressed:

cbetting@andrew.cmu.edu; km3b@andrew.cmu.edu; 
(a)

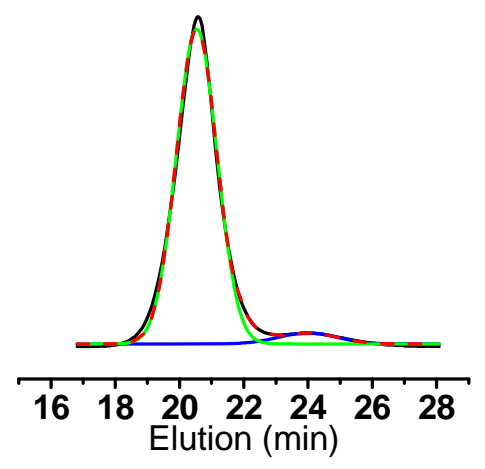

(c)

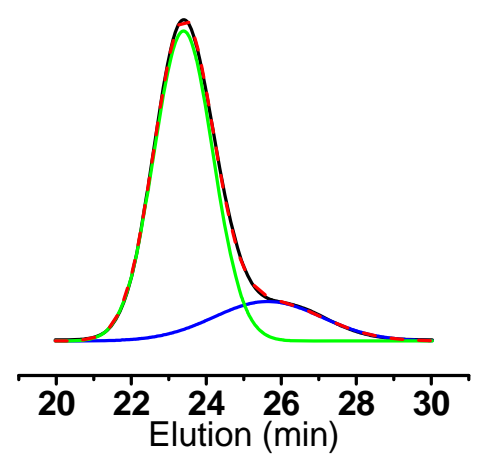

(e)

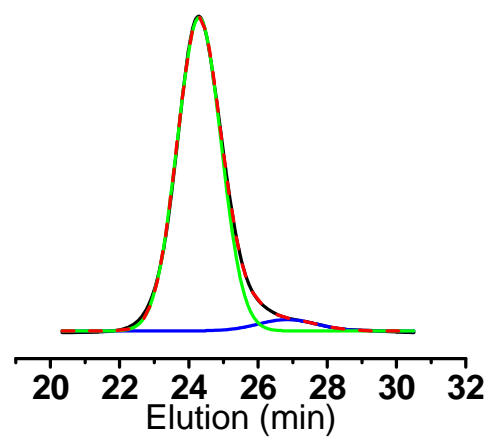

(b)

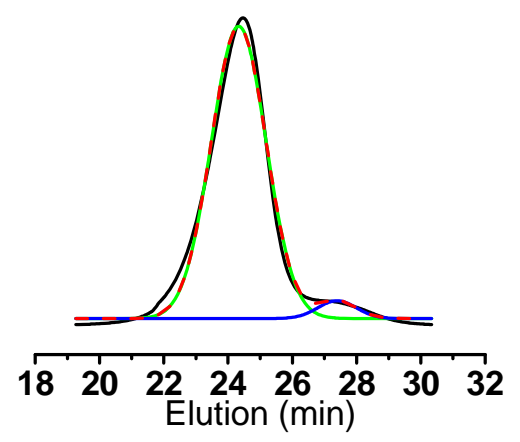

(d)

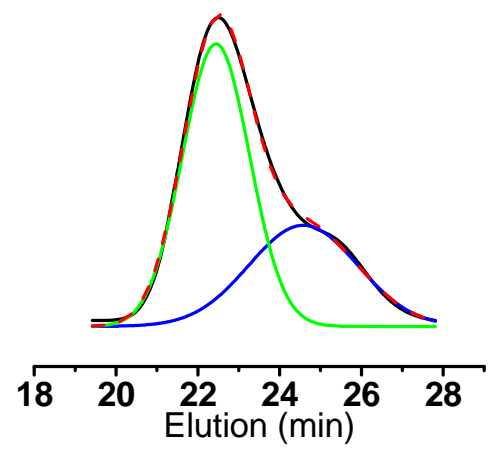

Figure S1. Multiple peak separation of star/MI from eluogram using MI of (a) PBA50- $b-\mathrm{P}_{t \mathrm{BA}} \mathrm{P}_{30}$

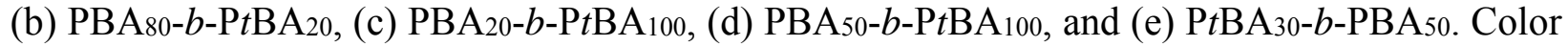
of curve: Black curve: original eluogram, blue: $\mathrm{MI}$, green: star, red (dashed): sum of green and blue. 
(a)

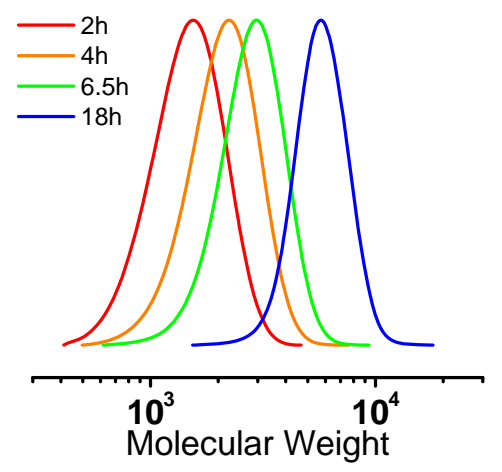

(c)

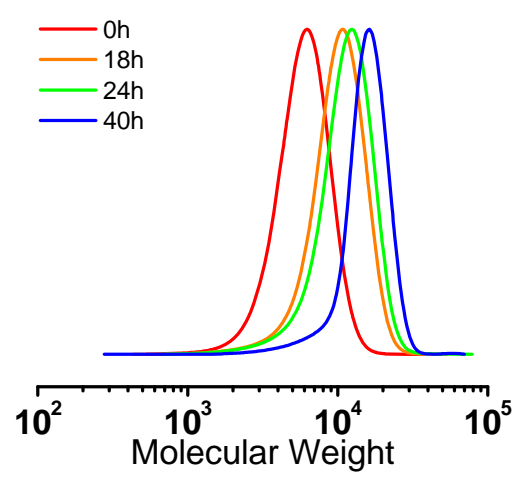

(b)

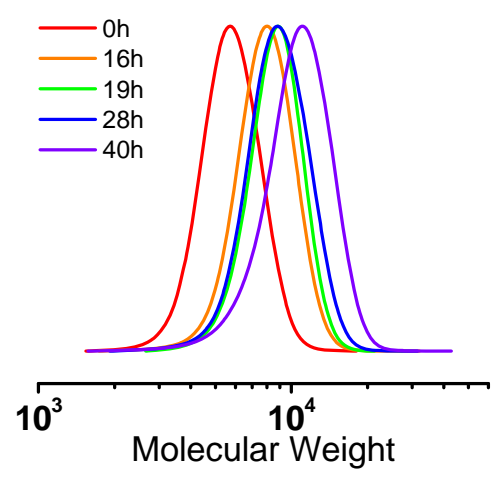

(d)

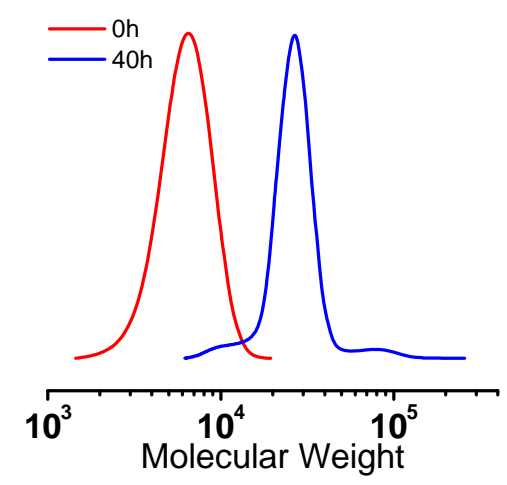

Figure S2. GPC traces during polymerization of (a) SAN with DP $=70$ and chain extension from PSAN 70 MIs with $t \mathrm{BA}$ for (b) PSAN $70-b-\mathrm{P} \mathrm{BA}_{40}$, (c) PSAN $70-b-\mathrm{P} \mathrm{BA}_{70}$, and (d) PSAN $70-b$ PtBA90 
(a)

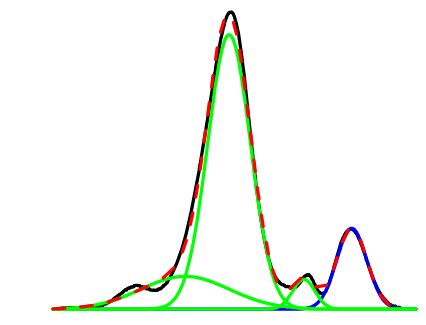

161820222426283032 Elution (min)

(c)

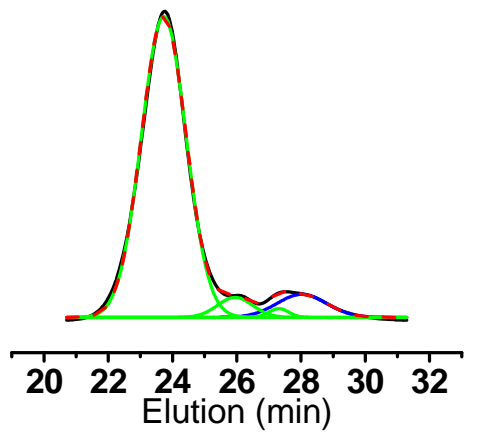

(e)

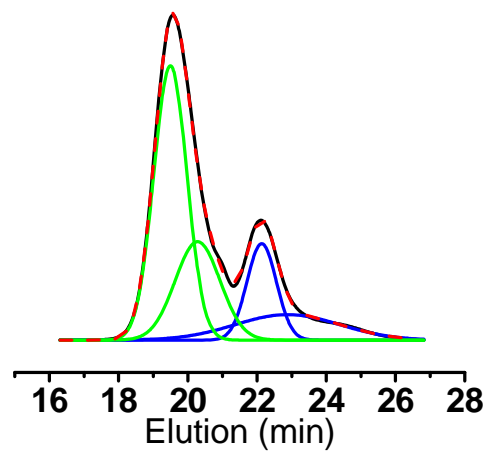

(b)

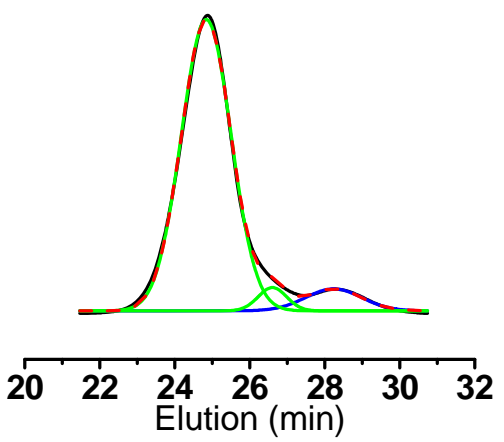

(d)

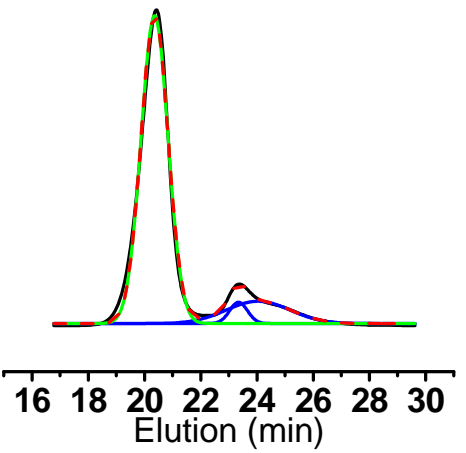

Figure S3. Multiple peak separation of star/MI from eluogram using MI of (a) PSAN $40-b-\mathrm{P} \mathrm{BA}_{24}$ (normal ATRP), (b) PSAN 40- $b-\mathrm{P} \mathrm{BA}_{24}$ (ARGET ATRP), (c) PSAN70- $b-\mathrm{P} t \mathrm{BA}_{40}$, (d) PSAN70- $b-$ PtBA70, and (e) PSAN70- $b-\mathrm{P} t \mathrm{BA} 90$. 
Table S1. Synthesis of PSAN-b-PtBA star polymers with different MIs. ${ }^{a}$

\begin{tabular}{|c|c|c|c|c|c|c|c|}
\hline entry & MI composition* & DP total & $M_{\mathrm{n}, \mathrm{MI}} \mathrm{C}^{\mathrm{C}}$ & $\begin{array}{l}M_{\mathrm{w}} / M_{\mathrm{n}}^{\mathrm{c}} \\
(\mathrm{arm})\end{array}$ & $M_{\mathrm{n}, \mathrm{star}}{ }^{\mathrm{c}}$ & $\begin{array}{l}M_{\mathrm{w}} / M_{\mathrm{n}}^{\mathrm{c}} \\
\text { (star) }\end{array}$ & $A_{\text {star }}{ }^{d}$ \\
\hline $1^{b}$ & $\mathrm{SAN}_{40}-b-t \mathrm{BA}_{24}$ & 64 & 3500 & 1.09 & 28700 & 1.98 & 0.85 \\
\hline 2 & $\mathrm{SAN}_{40}-b-t \mathrm{BA}_{24}$ & 64 & 3500 & 1.09 & 41300 & 1.39 & 0.94 \\
\hline 3 & $\mathrm{SAN}_{70}-b-t \mathrm{BA}_{40}$ & 110 & 10000 & 1.10 & 67800 & 1.49 & 0.90 \\
\hline 4 & $\mathrm{SAN}_{70-} b-t \mathrm{BA} 70$ & 140 & 14000 & 1.15 & 43500 & 1.72 & 0.87 \\
\hline 5 & $\mathrm{SAN}_{70}-b-t \mathrm{BA}_{90}$ & 160 & 17000 & 1.30 & 57000 & 2.19 & 0.69 \\
\hline
\end{tabular}

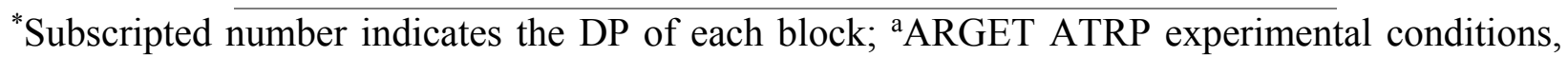
(except entry 1): $[\mathrm{MI}]_{0} /[\mathrm{DVB}]_{0} /\left[\mathrm{Sn}^{\mathrm{II}}(\mathrm{EH})_{2}\right]_{0} /\left[\mathrm{CuBr}_{2}\right]_{0} /\left[\mathrm{TPMA}_{0}=1 / 14 / 0.2 / 0.01 / 0.1\right.$, in anisole at $110^{\circ} \mathrm{C}$; bormal ATRP experimental conditions: $[\mathrm{MI}]_{0} /[\mathrm{DVB}]_{0} /[\mathrm{CuBr}]_{0} /[\mathrm{TPMA}]_{0}=1 / 14 / 0.8 / 0.8$, in anisole at $90^{\circ} \mathrm{C}$; ${ }^{\circ}$ Determined by THF GPC with RI detector (calibration with PSt standards); ${ }^{\mathrm{d}}$ Area fraction, determined by the multipeak splitting of the eluogram.

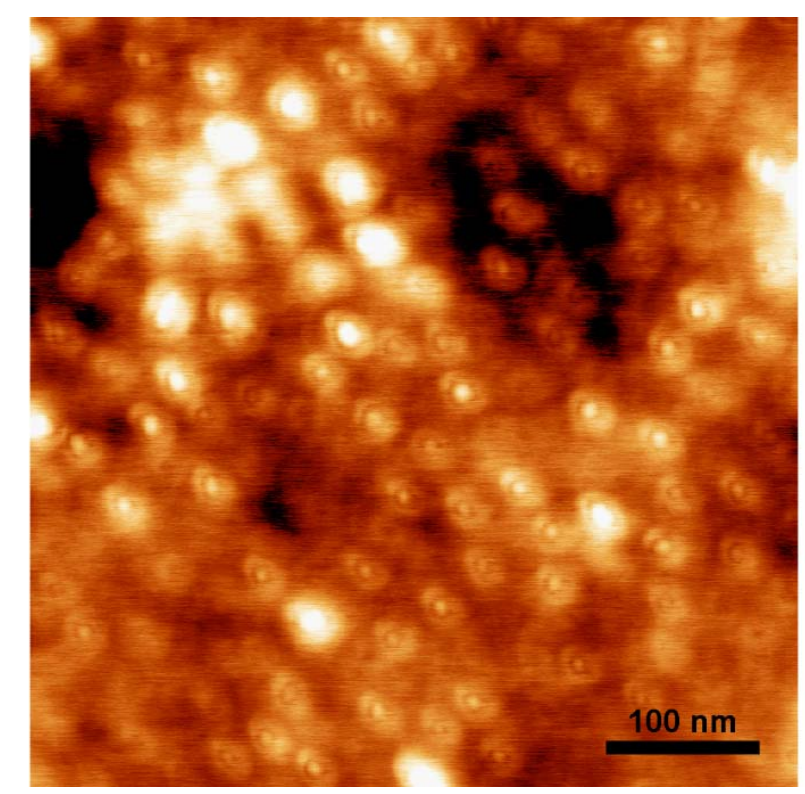

Figure S4. AFM image of the film composed of $\mathrm{SAN}_{70}-b-\mathrm{AA}_{40}$-star block copolymers. 